\title{
Status of Silicon in Rice (Oryza sativa L.) and its Correlation with Other Nutrients under Typic ustochrepts Soil
}

\author{
R.A. Patel ${ }^{1}$, K.C. Patel ${ }^{1}$ and J.K. Malav ${ }^{2 *}$ \\ ${ }^{1}$ Department of Agricultural Chemistry and Soil Science, B.A. College of Agriculture, \\ Anand Agricultural University, Anand-388 110, Gujarat, India \\ ${ }^{2}$ Department of Agricultural Chemistry and Soil Science, C.P. College of Agriculture, S. D. \\ Agricultural University, Sardarkrushinagar-385506, Gujarat, India \\ *Corresponding author
}

\section{A B S T R A C T}

\begin{tabular}{|l|}
\hline Ke y w o r d s \\
Silicon, Rice, \\
Nutrient status, \\
Correlation, \\
Nutrient ratio.
\end{tabular}

\section{Keymords}

The survey work was carried out in rice growing area of middle Gujarat region to assess the available Si content in rice and find out the relationship of Si content with plant. The silicon content of rice grain collected from the region ranged from 1.20 to 5.99 per cent with an average value of 3.01 per cent, respectively and the rice straw Si content varied from 3.52 to 9.80 per cent with an overall mean of 6.30 per cent of different districts. The total uptake of Si by rice was $88,114,87,91,73$ and $102 \mathrm{~kg} \mathrm{ha}^{-1}$ in different districts of middle Gujarat and the uptake of Si was nearly 2 to 45 times more as compared to N, P, K and $\mathrm{S}$ uptake by rice. The ratio of silicon and other nutrient contents in rice grain and straw indicated that amongst $\mathrm{N}, \mathrm{P}, \mathrm{K}, \mathrm{S}$ and $\mathrm{Na}$, the higher ratio was observed in $\mathrm{Si}$ : P indicating lower content of $\mathrm{P}$ in comparison with silicon. The correlation of coefficient (r) between $\mathrm{Si}$ content in rice and other nutrients indicated that $\mathrm{Si}$ content in rice grain and straw was positively correlated with $\mathrm{N}, \mathrm{P}, \mathrm{K}, \mathrm{Mn}$ and $\mathrm{Cu}$ contents and negatively correlated with $\mathrm{Fe}$ content of grain.

\section{Introduction}

Rice is heavy feeder of silicon, it's content as well as $\mathrm{Si} / \mathrm{Ca}$ ratio is higher than $1 \%$ and therefore, it requires high amounts of silicon for sustained productivity. Although agricultural soils are largely composed of silicate minerals, many soils contain an inadequate supply or are naturally low in plant available silicon. Most likely, silicon content in some regions might be limiting to sustainable rice production. In addition, silicon depletion can occur in traditional rice soils from the continuous monoculture of high yielding cultivars with intensive cultivation practices (Kawaguchi, 1966 and Miyake, 1993) especially if farmers are not applying the silicon removed by rice. The form of silicon absorbed by plant roots is silicic acid which is transported to the shoot. It is concentrated due to loss of water and is polymerized to colloidal silicic acid and finally to silica gel with increasing silicic acid concentration.

Rice is known as silicon accumulator and plant benefits much from silicon nutrition (Takahashi, 1995). Consequently, there is a 
definitive need to consider silicon as an agronomically essential element for increasing and /or sustaining rice production (Takahashi and Miyake, 1977). Silica is required for healthy and productive development of the rice plant (Yoshida, 1975). This element is absorbed by rice from the soil in large amounts that is several fold greater than those of other macro- nutrients. On an average, it is estimated that a rice crop producing a total grain yield of about 5 tonnes $\mathrm{ha}^{-1}$ will normally remove 230 to $470 \mathrm{Si} \mathrm{kg}$ $\mathrm{ha}^{-1}$ (500-1000 $\mathrm{SiO}_{2} \mathrm{~kg} \mathrm{ha}^{-1}$ ) from soil (Savant et al., 1997). Recent research and advances in physiology, biochemistry and genetics suggest that silicon interacts with other native or applied nutrients and has the potential to induce resistance/ tolerance in the rice plant to biotic (insects, pests and diseases) and abiotic stresses like $\mathrm{Al}, \mathrm{Fe}$ and $\mathrm{Mn}$ toxicity; salt injury, lodging, etc. (Epstein, 1994). Thus, by and large, there is a general consensus that silicon is a quassi-essential element for rice plant.

Silicon is absorbed by rice from the soil in large amounts that is several fold greater than those of other macro- nutrients. On an average, it is estimated that a rice crop producing a total grain yield of about 5 tonnes $\mathrm{ha}^{-1}$ will normally remove 230 to $470 \mathrm{~kg} \mathrm{Si}$ ha $^{-1}$ (500-1000 kg SiO $\mathrm{ha}^{-1}$ ) from soil (Savant et al., 1997). In Middle Gujarat, total area under rice cultivation is 4.30 lakh ha with total production of 7.20 lakh M. T. and productivity of $1540 \mathrm{~kg} \mathrm{ha}^{-1}$ during 2008-09 (Anon., 2009). But, there is a progressive decline in yield in rice ecosystems may be due to the depletion of plant available silicon in soil. Hence, considering this problem, it was felt essential to identify soil and plant silicon profiles within intensively cultivated rice ecosystems throughout middle Gujarat region as till date the issue of silicon nutrition in rice production remains largely unexplored. By identifying and implementing ideal silicon nutrition management strategies, declining yield trends in many rice production systems could be reversed. Therefore, the paper deals with status of silicon in rice (Oryza sativa) and its correlation with other nutrients under Typic Ustochrepts soil.

\section{Materials and Methods}

\section{Collection of rice samples}

Twenty representative samples from preidentified 20 locations in each district were collected after harvest of rice crop in the month of October- November in 2009 and analyzed as per standard methods.

\section{Method of plant analysis}

Nitrogen, phosphorous, potassium and micronutrients ( $\mathrm{Fe}, \mathrm{Mn}, \mathrm{Zn}$ and $\mathrm{Cu}$ ) were estimated by standard method suggested by Jackson (1973). Sulphur was analyzed by turbidimetric method as given by Chaudhari and Cornfield (1966). Silicon from plant was estimated by colorimetric method as per Dai et al., (2005).

\section{Statistical analysis}

Simple correlation coefficients of silicon with physical and chemical properties of soils and nutrient contents of plants were worked out by multiple regression technique (Steel and Torrie, 1982).

\section{Results and Discussion}

\section{Silicon content in rice}

The grain and plant samples each of analysed for silicon content and result given in Table 1 showed that the silicon content of rice grain in middle Gujarat region was ranged from 1.20 to 5.99 per cent with an average value of 3.01 per cent, respectively and the rice straw silica 
content varied from 3.52 to 9.80 per cent with an overall mean of 6.30 percent of different districts of middle Gujarat region. The district wise mean silicon content of rice grain was 2.43, 4.89, 3.28, 2.13, 1.81 and 3.45 per cent, respectively. The average silicon content of rice straw of different districts was 6.40, 6.53, $5.40,6.98,5.54$ and 6.76 per cent, respectively. The lower value of Si uptake by grain and straw in Dahod districts was mainly due to the lower availability of $\mathrm{Si}$ as compared to other districts. Similar ranges for $\mathrm{Si}$ content of rice grain and straw were reported by many researchers. Nayar et al., (1977) reported that silicon content in harvested rice straw and grain was 7.13 and 2.67 per cent, respectively. The $\mathrm{SiO}_{2}$ content of rice straw ranged from 4.4 to 19.6 per cent with an average of 11.0 per cent (Yoshida, 1981) and likewise, Si contents in harvested rice grain and straw were 3.35 and 9.40 per cent, respectively (Nayar et al., 1975).

The categorization of 120 soil samples was done as per available $\mathrm{Si}$ status in different categories viz., <25.0, $25-50,50-75$ and > $75 \mathrm{mg} \mathrm{Si} \mathrm{kg}{ }^{-1}$ soil. The data given in Table 2 showed that around 60 per cent soils of different districts had less than $50.0 \mathrm{mg}$ Si $\mathrm{kg}^{-1}$ and on hectare basis; it was 2.93 lakh hectares of rice growing areas of middle Gujarat region. The lower available Si status may be attributed to the adoption of integrated nutrient management systems (INMS) for sustainable rice production, wherein silicon was not a part of INMS.

\section{Nutrients content in rice}

From the six districts of middle Gujarat region of rice growing areas, rice grain and straw samples were also collected along with soil samples and analyzed for their silicon content by colorimetric method (Dai et al., 2005). The data given in Table 2 revealed that the overall silicon content in rice grain ranged from 1.20 to 5.99 per cent with mean of 3.01 per cent and the silicon content in rice straw ranged from 3.52 to 9.80 per cent with mean of 6.30 per cent. The silicon content in rice grain was found higher in Kheda district ranged from 3.58 to 5.78 per cent with mean of 4.89 per cent and lowest was found in Dahod district ranged from 1.28 to 3.02 per cent with mean of 1.81 per cent. The mean silicon content of rice grain of different districts was $2.43,4.89,3.28,2.13,1.81$ and 3.45 per cent, respectively. The lowest content of $\mathrm{Si}$ in rice grain resulted due to low availability of silicon in soils of Dahod district and reverse in Kheda district where soil contain high available silicon. Silicon content in rice straw was found high in Panchmahals district ranged from 5.01 to 9.27 per cent with mean of 6.98 per cent. The silicon content in rice straw was found lower in Anand district ranged from 3.52 to 7.21 per cent with mean of 5.40 per cent. The average silicon content of rice straw of different districts was 6.40, 6.53, 5.40, 6.98, 5.54 and 6.76 per cent, respectively (Table 3 ).

The collected rice grain samples were also analyzed for important nutrient elements viz., $\mathrm{N}, \mathrm{P}, \mathrm{K}, \mathrm{S}$ and $\mathrm{Na}$ and presented in Table 2. The analytical results revealed that the average content of nitrogen in rice grain samples was $1.32,1.40,1.15,1.37,1.33$ and 1.11 per cent respectively in different districts of middle Gujarat region. Whereas mean $\mathrm{P}$ content were $0.13,0.10,0.09,0.10,0.08$ and 0.11 per cent, respectively. The average content of K was found $0.21,0.27,0.19,0.18$, 0.19 and 0.27 per cent, respectively. The mean S content was $0.27,0.12,0.20,0.13$, 0.16 and 0.14 per cent respectively among different districts. Whereas mean content of $\mathrm{Na}$ in rice grain samples was $0.10,0.08,0.07$, $0.07,0.06$ and 0.07 per cent respectively in rice grain samples of different districts of middle Gujarat region. The overall mean content of $\mathrm{N}, \mathrm{P}, \mathrm{K}, \mathrm{S}$ and $\mathrm{Na}$ in grain was 
$1.28,0.09,0.22,0.17$ and 0.08 per cent, respectively. The data of nutrients content in rice grain showed that the $\mathrm{Si}$ accumulation was higher than other nutrients. The silicon content in grain was around double than N, 30 times more than $\mathrm{P}$ and $\mathrm{Na}$, while 15 times more than $\mathrm{K}$ and $\mathrm{S}$ content in rice grain.

The rice straw samples were analyzed for important nutrient elements viz., N, P, K, S and $\mathrm{Na}$ and their contents were presented in Table 3 . The analytical results indicated that the average content of nitrogen in rice straw samples was 3.05, 2.21, 2.92, 2.50, 2.74 and 2.42 per cent, respectively in different districts of middle Gujarat region. Whereas mean $\mathrm{P}$ content was $0.14,0.12,0.10,0.10$, 0.20 and 0.19 per cent, respectively. The average content of $\mathrm{K}$ was found $0.22,0.32$, $0.36,0.37,0.31$ and 0.44 per cent, respectively. The mean $\mathrm{S}$ content was 0.47 , $0.66,0.36,0.66,0.60$ and 0.76 per cent, respectively among different districts. The overall $\mathrm{N}, \mathrm{P}, \mathrm{K}, \mathrm{S}$ and $\mathrm{Na}$ content in rice straw ranged from 1.82-3.58, 0.05-0.29, 0.19-0.49, 0.1-1.01 and 0.13-0.44 per cent, respectively.

Remarkable differences found in the micronutrient content of rice grain given in Table 2. Analytical results showed that the highest value of Fe was found in the Anand district $\left(663 \mathrm{mg} \mathrm{kg}^{-1}\right)$ and minimum $(28 \mathrm{mg}$ $\mathrm{kg}^{-1}$ ) was found in Kheda district. The average content of $\mathrm{Fe}$ was found 170, 301, 291, 218, 200, $148 \mathrm{mg} \mathrm{kg}^{-1}$ in different districts. Average Mn content was found 30, $61,49,22,35,79 \mathrm{mg} \mathrm{kg}^{-1}$ while mean $\mathrm{Zn}$ content was observed 19, 31, 33, 24, 30, 23 $\mathrm{mg} \mathrm{kg}^{-1}$ respectively in the different districts of middle Gujarat region. The $\mathrm{Cu}$ content was ranged from 3.0 to $21.0 \mathrm{mg} \mathrm{kg}^{-1}$ in rice grain with mean value $7.0 \mathrm{mg} \mathrm{kg}^{-1}$.

The rice straw samples were also analyzed for micronutrients content and data presented in Table 3. The average Fe content of rice straw samples was 259, 387, 401, 270, 399 and 300 $\mathrm{mg} \mathrm{kg}^{-1}$, respectively in different districts of middle Gujarat. The mean Mn content was found 166, 189, 113, 254, 189 and $316 \mathrm{mg}$ $\mathrm{kg}^{-1}$, respectively. The average $\mathrm{Zn}$ content of straw samples was 30, 21, 39, 30, 32 and 31 $\mathrm{mg} \mathrm{kg}{ }^{-1}$, respectively. Whereas, the average $\mathrm{Cu}$ content of straw samples was $6,10,10,5$, 8 and $7 \mathrm{mg} \mathrm{kg}^{-1}$, respectively. The overall mean content of $\mathrm{Fe}, \mathrm{Mn}, \mathrm{Zn}$ and $\mathrm{Cu}$ was 401, 238, 30 and $8.0 \mathrm{mg} \mathrm{kg}^{-1}$, respectively. Similar ranges for $\mathrm{Si}$ content of rice grain and straw were reported by many researchers. Nayar et al., (1977) reported that silicon content in harvested straw and grain was 7.13 and 2.67 per cent, respectively. The $\mathrm{SiO}_{2}$ content of straw ranged from 4.4 to 19.6 per cent with an average of 11.0 per cent (Imaizumi and Yoshida, 1958) and likewise, Si contents in harvested grain and straw were 3.35 and 9.40 per cent, respectively (Nayar et al., 1975).

\section{Uptake of silicon and other nutrients by rice}

The results of nutrients uptake by of rice grain and straw in different districts of middle Gujarat was reported in Table 4. The average silicon uptake by rice grain was 23.6, 48.9, $32.8,21.1,18.1,34.5 \mathrm{~kg} \mathrm{ha}^{-1}$ and 64.0, 65.3, $54.0,69.8,55.4$ and $67.6 \mathrm{~kg} \mathrm{ha}^{-1}$ by straw in Ahmedabad, Kheda, Anand, Panchmahals, Dahod and Vadodara, respectively. The lower value of $\mathrm{Si}$ uptake by grain and straw in Dahod districts resulted due to availability of $\mathrm{Si}$ was low in soils as compared different districts. The maximum total uptake of $\mathrm{Si}$ recorded as $114.2 \mathrm{~kg} \mathrm{ha}^{-1}$ in the Kheda districts and minimum as $73.4 \mathrm{~kg} \mathrm{ha}^{-1}$ in the Dahod district. The average total uptake of silicon by rice was 87.6, 114.2, 86.8, 90.9, $73.4,102.1 \mathrm{~kg} \mathrm{ha}^{-1}$ in the different districts of middle Gujarat region (Table 6).

The uptake pattern of $\mathrm{N}, \mathrm{P}, \mathrm{K}, \mathrm{S}$ and $\mathrm{Na}$ by rice grain was in descending order: $\mathrm{N}>\mathrm{K}>\mathrm{S}$ 
> P > Na. The uptake of Si was nearly 2 to 20 times more as compared to major nutrients uptake which indicated that more requirement of silicon by rice as compared to others nutrients. The overall mean uptake of $\mathrm{N}, \mathrm{P}, \mathrm{K}$, $\mathrm{S}$ and $\mathrm{Na}$ by rice grain was $12.7,1.04,2.17$, 1.71 and $0.73 \mathrm{~kg} \mathrm{ha}^{-1}$, respectively (Table 4). The $\mathrm{SiO}_{2}$ uptake or content of the plants might be increased to some extent by applying silicate material. Similar results were also reported by Ganaie et al., (2015) and Korndorfer et al., (2001) in some soils of Florida and Meshram et al., (2015) in soils of Sri Lanka, Singh et al., (2006) in soils of India.

The Si uptake by straw was nearly two times more than $\mathrm{N}$ uptake and 40,20 and 12 times more as compared to $\mathrm{P}, \mathrm{K}$ and $\mathrm{S}$ uptake by rice straw, respectively (Table 5). The overall mean uptake of $\mathrm{Fe}, \mathrm{Mn}, \mathrm{Zn}$ and $\mathrm{Cu}$ in rice grain was $220.8,45.9,26.5$ and $6.91 \mathrm{~g} \mathrm{ha}^{-1}$, respectively in the different districts of middle Gujarat region. Because of higher available $\mathrm{Fe}$ the uptake of $\mathrm{Fe}$ by rice grain was also found higher as compared to other micro nutrients (Table 4). The average micronutrients ( $\mathrm{Fe}, \mathrm{Mn}, \mathrm{Zn}$ and $\mathrm{Cu}$ ) uptake by rice straw was 336.1, 204.6, 30.4 and $7.6 \mathrm{~g}$ ha 1 in different districts of middle Gujarat region, respectively (Table 5). The total uptake of silicon by rice was nearly $2,45,18$, 13 and 30 times more than uptake of N, P, K, $\mathrm{S}$ and $\mathrm{Na}$, respectively in middle Gujarat region (Table 6). The average uptake of $\mathrm{Fe}$, $\mathrm{Mn}, \mathrm{Zn}$ and $\mathrm{Cu}$ by rice was 645.0, 245.2, 56.9 and $14.4 \mathrm{~g} \mathrm{ha}^{-1}$, respectively in different districts.

The results obtained for different nutrients present in rice grain and straw with respect to $\mathrm{Si}$ can be supported by many research findings. Okuda and Takahashi (1962 b) noticed that the added silicon increased the translocation rate of absorbed phosphorous to the grain especially at the phosphorus deficient level. This effect might have resulted from the action of silicon, in which the increasing amount of silicon decreased the iron content of rice plant and increased the phosphorous / iron ratio in the plant, especially at the phosphorus deficient level.

From these experiments, it concluded that silicon seems to retard the excessive uptake of phosphorous by rice to promote the translocation of phosphorous in rice plant. Islam and Saha (1969) inferred that the application of silicon generally decreased the nitrogen, protein and potassium content of rice plants. Its application also decreased the iron and manganese contents. The use of silicon resulted in an increase in phosphorous, calcium, magnesium, silicon and carbohydrate contents of rice plant.

Analysis of straw by Takijima et al., (1970) showed that addition of dolomite slag lowered $\mathrm{N}$ and $\mathrm{Fe}$ and raised the contents of $\mathrm{P}, \mathrm{K}$ and $\mathrm{Mg}$ as well as $\mathrm{Si}$. According to Elawad and Green (1979) due to synergistic effect, applied silicon had the potential to raise optimum rate of $\mathrm{N}$, thus helping to enhance yield due to $\mathrm{N}$ inputs. Cheng (1982) informed that yield response over the control would not be obtained if available silicon exceeded 11 $\mathrm{SiO}_{2} \mathrm{mg} 100 \mathrm{~g}^{-1}$ in the soil. There was an increase in $\mathrm{Ca}, \mathrm{Mg}, \mathrm{Si}$ and carbohydrate contents after the addition of silicon to the culture solution@75 ppm silicon which also decreased the accumulation of $\mathrm{Mn}, \mathrm{Fe}, \mathrm{Zn}$, $\mathrm{Cu}, \mathrm{N}, \mathrm{P}$ and transpiration rate. It is concluded that addition of silicon is particularly effective when combined with heavy doses of nitrogen and magnesium. Ma and Takahashi (1989) observed that both content and uptake of phosphorous were decreased by the addition of silicic acid and the effect was more pronounced at the vegetative stage than at the reproductive stage. Verma and Minhas (1989) indicated uptake of Mn was not affected by silicon addition. 
Table.1 Status of available silicon content in rice in different districts of middle Gujarat

\begin{tabular}{|c|c|c|c|c|c|c|c|}
\hline \multirow{2}{*}{ Sr. No. } & \multirow{2}{*}{ District } & \multirow{2}{*}{$\begin{array}{c}\text { No. of Taluka } \\
\text { covered }\end{array}$} & \multirow{2}{*}{ No. of sample } & \multicolumn{2}{c|}{ Grain Si (\%) } & \multicolumn{2}{c|}{ Straw Si (\%) } \\
\cline { 5 - 8 } & & & Range & Mean & Range & Mean \\
\hline 1 & Ahmedabad & 5 & 20 & $1.20-5.99$ & 2.43 & $4.97-8.63$ & 6.40 \\
\hline 2 & Kheda & 3 & 20 & $3.58-5.78$ & 4.89 & $4.30-9.48$ & 6.53 \\
\hline 3 & Anand & 5 & 20 & $1.40-5.44$ & 3.28 & $3.52-7.21$ & 5.40 \\
\hline 4 & Panchmahals & 3 & 20 & $1.22-3.58$ & 2.13 & $5.01-9.27$ & 6.98 \\
\hline 5 & Dahod & 2 & 20 & $1.28-3.02$ & 1.81 & $4.19-7.53$ & 5.54 \\
\hline 6 & Vadodara & 3 & 20 & $1.92-5.78$ & 3.45 & $3.84-9.80$ & 6.76 \\
\hline \multicolumn{2}{|c|}{ Overall } & & $\mathbf{1 2 0}$ & $\mathbf{1 . 2 0 - 5 . 9 9}$ & $\mathbf{3 . 0 1}$ & $\mathbf{3 . 5 2 - 9 . 8 0}$ & $\mathbf{6 . 3 0}$ \\
\hline
\end{tabular}

Table. 2 Nutrients content in rice grain in different districts of middle Gujarat

\begin{tabular}{|c|c|c|c|c|c|c|c|c|c|c|}
\hline \multirow{2}{*}{ Sr. No. } & \multirow{2}{*}{ District } & $\mathbf{N}$ & $\mathbf{P}$ & $\mathbf{K}$ & $\mathbf{S}$ & $\mathbf{N a}$ & Fe & Mn & $\mathbf{Z n}$ & $\mathrm{Cu}$ \\
\hline & & \multicolumn{5}{|c|}{$(\%)$} & \multicolumn{4}{|c|}{$\left(\mathrm{mg} \mathrm{kg}^{-1}\right)$} \\
\hline 1 & Ahmedabad & $\begin{array}{c}1.06-1.62 \\
(1.32)\end{array}$ & $\begin{array}{c}0.03-0.21 \\
(0.13)\end{array}$ & $\begin{array}{c}0.15-0.28 \\
(0.21)\end{array}$ & $\begin{array}{c}0.15-0.39 \\
(0.27)\end{array}$ & $\begin{array}{c}0.06-0.16 \\
(0.10)\end{array}$ & $\begin{array}{c}121-228 \\
(170)\end{array}$ & $\begin{array}{c}17-42 \\
(30)\end{array}$ & $\begin{array}{c}11-24 \\
(19)\end{array}$ & $\begin{array}{l}3-10 \\
(5.0)\end{array}$ \\
\hline 2 & Kheda & $\begin{array}{c}1.20-1.60 \\
(1.40)\end{array}$ & $\begin{array}{c}0.03-0.18 \\
(0.11)\end{array}$ & $\begin{array}{c}0.21-0.30 \\
(0.27)\end{array}$ & $\begin{array}{c}0.06-0.21 \\
(0.12)\end{array}$ & $\begin{array}{c}0.06-0.11 \\
(0.08)\end{array}$ & $\begin{array}{c}28-576 \\
(301)\end{array}$ & $\begin{array}{c}15-124 \\
(61)\end{array}$ & $\begin{array}{c}16-68 \\
(31)\end{array}$ & $\begin{array}{l}\text { 4-16 } \\
(8.0)\end{array}$ \\
\hline 3 & Anand & $\begin{array}{c}0.87-1.43 \\
(1.15)\end{array}$ & $\begin{array}{c}0.07-0.12 \\
(0.09)\end{array}$ & $\begin{array}{c}0.16-0.28 \\
(0.19)\end{array}$ & $\begin{array}{c}0.10-0.30 \\
(0.20)\end{array}$ & $\begin{array}{c}0.05-0.08 \\
(0.07)\end{array}$ & $\begin{array}{c}78-663 \\
(291)\end{array}$ & $\begin{array}{c}21-83 \\
(49)\end{array}$ & $\begin{array}{c}20-66 \\
(33)\end{array}$ & $\begin{array}{l}4-21 \\
(9.0)\end{array}$ \\
\hline 4 & Panchmahals & $\begin{array}{c}1.06-1.65 \\
(1.37)\end{array}$ & $\begin{array}{c}0.07-0.17 \\
(0.10)\end{array}$ & $\begin{array}{c}0.15-0.26 \\
(0.18)\end{array}$ & $\begin{array}{c}0.06-0.25 \\
(0.13)\end{array}$ & $\begin{array}{c}0.04-0.09 \\
(0.07)\end{array}$ & $\begin{array}{c}42-661 \\
(218)\end{array}$ & $7-37(22)$ & $9-41(24)$ & $\begin{array}{l}3-18 \\
(7.0)\end{array}$ \\
\hline 5 & Dahod & $\begin{array}{c}1.15-1.48 \\
(1.33)\end{array}$ & $\begin{array}{c}0.04-0.10 \\
(0.08)\end{array}$ & $\begin{array}{c}0.15-0.30 \\
(0.19)\end{array}$ & $\begin{array}{c}0.07-0.28 \\
(0.16)\end{array}$ & $\begin{array}{c}0.05-0.07 \\
(0.06)\end{array}$ & $\begin{array}{c}30-483 \\
(200)\end{array}$ & $\begin{array}{c}19-78 \\
(35)\end{array}$ & $\begin{array}{c}20-46 \\
(30)\end{array}$ & $\begin{array}{l}3-12 \\
(7.0)\end{array}$ \\
\hline 6 & Vadodara & $\begin{array}{c}0.84-1.37 \\
(1.11)\end{array}$ & $\begin{array}{c}0.03-0.21 \\
(0.11)\end{array}$ & $\begin{array}{c}0.17-0.33 \\
(0.27)\end{array}$ & $\begin{array}{c}0.08-0.23 \\
(0.14)\end{array}$ & $\begin{array}{c}0.06-0.08 \\
(0.07)\end{array}$ & $\begin{array}{c}37-531 \\
(148)\end{array}$ & $\begin{array}{c}41-162 \\
(79)\end{array}$ & $\begin{array}{c}12-36 \\
(23)\end{array}$ & $\begin{array}{l}3-10 \\
(6.0)\end{array}$ \\
\hline \multicolumn{2}{|c|}{ Overall } & $\begin{array}{c}0.84-1.65 \\
(1.28)\end{array}$ & $\begin{array}{c}0.03-0.21 \\
(0.09)\end{array}$ & $\begin{array}{c}0.15-0.33 \\
(0.22)\end{array}$ & $\begin{array}{c}0.06-0.39 \\
(0.17) \\
\end{array}$ & $\begin{array}{c}0.04-0.16 \\
(0.08)\end{array}$ & $\begin{array}{c}28-663 \\
(221)\end{array}$ & $\begin{array}{c}7-162 \\
(46)\end{array}$ & $9-68(27)$ & $\begin{array}{l}3-21 \\
(7.0)\end{array}$ \\
\hline
\end{tabular}


Table.3 Nutrients content in rice straw in different districts of middle Gujarat

\begin{tabular}{|c|c|c|c|c|c|c|c|c|c|c|}
\hline \multirow{2}{*}{ Sr. No. } & \multirow{2}{*}{ District } & $\mathbf{N}$ & $\mathbf{P}$ & $\mathbf{K}$ & $\mathbf{S}$ & $\mathbf{N a}$ & $\mathbf{F e}$ & Mn & $\mathbf{Z n}$ & $\mathbf{C u}$ \\
\hline & & \multicolumn{5}{|c|}{$(\%)$} & \multicolumn{4}{|c|}{$\left(\mathrm{mg} \mathrm{kg}^{-1}\right)$} \\
\hline 1 & Ahmedabad & $\begin{array}{c}2.74-3.58 \\
(3.05)\end{array}$ & $\begin{array}{c}0.05-0.22 \\
(0.14)\end{array}$ & $\begin{array}{c}0.19-0.29 \\
(0.22)\end{array}$ & $\begin{array}{c}0.25-0.74 \\
(0.47)\end{array}$ & $\begin{array}{c}0.22-0.44 \\
(0.33)\end{array}$ & $\begin{array}{c}72-487 \\
(259)\end{array}$ & $\begin{array}{c}51-289 \\
(166)\end{array}$ & $11-46(30)$ & $2-10(6.0)$ \\
\hline 2 & Kheda & $\begin{array}{c}1.82-2.74 \\
(2.21)\end{array}$ & $\begin{array}{c}0.09-0.19 \\
(0.12)\end{array}$ & $\begin{array}{c}0.29-0.37 \\
(0.32)\end{array}$ & $\begin{array}{c}0.31-0.93 \\
(0.66)\end{array}$ & $\begin{array}{c}0.23-0.31 \\
(0.26)\end{array}$ & $\begin{array}{c}66-626 \\
(387)\end{array}$ & $\begin{array}{c}66-513 \\
(189)\end{array}$ & $4-35(21)$ & $\begin{array}{c}5-18 \\
(10.0)\end{array}$ \\
\hline 3 & Anand & $\begin{array}{c}2.49-3.58 \\
(2.92)\end{array}$ & $\begin{array}{c}0.07-0.13 \\
(0.10)\end{array}$ & $\begin{array}{c}0.31-0.40 \\
(0.36)\end{array}$ & $\begin{array}{c}0.19-0.48 \\
(0.36)\end{array}$ & $\begin{array}{c}0.14-0.26 \\
(0.19)\end{array}$ & $\begin{array}{c}33-761 \\
(401)\end{array}$ & $\begin{array}{c}28-289 \\
(113)\end{array}$ & 21-66 (39) & $\begin{array}{c}3-17 \\
(10.0)\end{array}$ \\
\hline 4 & Panchmahals & $\begin{array}{c}1.99-3.11 \\
(2.50)\end{array}$ & $\begin{array}{c}0.07-0.17 \\
(0.10)\end{array}$ & $\begin{array}{c}0.32-0.41 \\
(0.37)\end{array}$ & $\begin{array}{c}0.25-0.85 \\
(0.66)\end{array}$ & $\begin{array}{c}0.13-0.33 \\
(0.24)\end{array}$ & $\begin{array}{c}53-687 \\
(270)\end{array}$ & $\begin{array}{c}67-684 \\
(254)\end{array}$ & $15-55(30)$ & $1-11(5.0)$ \\
\hline 5 & Dahod & $\begin{array}{c}2.32-3.11 \\
(2.74)\end{array}$ & $\begin{array}{c}0.10-0.29 \\
(0.20)\end{array}$ & $\begin{array}{c}0.25-0.39 \\
(0.31)\end{array}$ & $\begin{array}{c}0.24-0.89 \\
(0.60)\end{array}$ & $\begin{array}{c}0.22-0.31 \\
(0.27)\end{array}$ & $\begin{array}{c}181-869 \\
(399)\end{array}$ & $\begin{array}{c}125-323 \\
(189)\end{array}$ & $17-48(32)$ & $3-18(8.0)$ \\
\hline 6 & Vadodara & $\begin{array}{c}2.24-2.60 \\
(2.42)\end{array}$ & $\begin{array}{c}0.13-0.23 \\
(0.19)\end{array}$ & $\begin{array}{c}0.40-0.49 \\
(0.44)\end{array}$ & $\begin{array}{c}0.57-1.01 \\
(0.76)\end{array}$ & $\begin{array}{c}0.13-0.22 \\
(0.18)\end{array}$ & $\begin{array}{c}151-614 \\
(300)\end{array}$ & $\begin{array}{c}113-629 \\
(316)\end{array}$ & $15-65(31)$ & $2-15(7.0)$ \\
\hline \multicolumn{2}{|c|}{ Overall } & $\begin{array}{c}1.82-3.58 \\
(2.64)\end{array}$ & $\begin{array}{c}0.05-0.29 \\
(0.14)\end{array}$ & $\begin{array}{c}0.19-0.49 \\
(0.34)\end{array}$ & $\begin{array}{c}0.10-1.01 \\
(0.56)\end{array}$ & $\begin{array}{c}0.13-0.44 \\
(0.25)\end{array}$ & $\begin{array}{c}33-869 \\
(401)\end{array}$ & $\begin{array}{c}28-684 \\
(238)\end{array}$ & 4-66 (30) & $1-18(8.0)$ \\
\hline
\end{tabular}

Table.4 Nutrients uptake by one tonne of rice grain in different districts of middle Gujarat

\begin{tabular}{|c|c|c|c|c|c|c|c|c|c|c|}
\hline \multirow{2}{*}{$\begin{array}{l}\text { Sr. } \\
\text { No. }\end{array}$} & \multirow{2}{*}{ District } & \multicolumn{5}{|c|}{ Grain uptake $\left(\mathrm{kg} \mathrm{ha}^{-1}\right)$} & \multicolumn{4}{|c|}{ Grain uptake (g ha $\left.{ }^{-1}\right)$} \\
\hline & & $\mathbf{N}$ & $\mathbf{P}$ & $\mathbf{K}$ & $\mathbf{S}$ & $\mathbf{N a}$ & $\mathbf{F e}$ & Mn & $\mathbf{Z n}$ & $\mathbf{C u}$ \\
\hline 1 & Ahmedabad & $\begin{array}{c}10.6-16.2 \\
(13.2)\end{array}$ & $\begin{array}{c}0.32-2.09 \\
(1.30)\end{array}$ & $\begin{array}{c}1.45-2.76 \\
(2.06)\end{array}$ & $\begin{array}{c}1.46-3.87 \\
(2.73)\end{array}$ & $\begin{array}{c}0.55-1.62 \\
(1.01)\end{array}$ & $\begin{array}{l}72-487 \\
(169.6)\end{array}$ & $\begin{array}{l}17-42 \\
(30.4) \\
\end{array}$ & $\begin{array}{l}11-24 \\
(18.6) \\
\end{array}$ & $3-10(5.4)$ \\
\hline 2 & Kheda & $\begin{array}{c}12.0-16.5 \\
(14.0)\end{array}$ & $\begin{array}{c}0.28-2.13 \\
(1.15)\end{array}$ & $\begin{array}{c}2.06-3.02 \\
(2.68)\end{array}$ & $\begin{array}{c}0.61-2.09 \\
(1.24)\end{array}$ & $\begin{array}{c}0.58-0.76 \\
(0.66)\end{array}$ & $\begin{array}{l}28-576 \\
(300.9)\end{array}$ & $\begin{array}{c}15-124 \\
(61.0)\end{array}$ & $\begin{array}{l}16-68 \\
(30.9)\end{array}$ & $4-16(7.8)$ \\
\hline 3 & Anand & $\begin{array}{c}8.70-14.3 \\
(11.5)\end{array}$ & $\begin{array}{c}0.71-1.32 \\
(0.91)\end{array}$ & $\begin{array}{c}1.56-2.83 \\
(1.92)\end{array}$ & $\begin{array}{c}0.98-2.97 \\
(1.95)\end{array}$ & $\begin{array}{c}0.45-0.81 \\
(0.68)\end{array}$ & $\begin{array}{l}78-663 \\
(291.1)\end{array}$ & $\begin{array}{l}21-83 \\
(48.6)\end{array}$ & $\begin{array}{l}20-66 \\
(33.2)\end{array}$ & $4-21(8.5)$ \\
\hline 4 & Panchmahals & $\begin{array}{c}10.6-16.5 \\
(13.7)\end{array}$ & $\begin{array}{c}0.72-1.76 \\
(1.07)\end{array}$ & $\begin{array}{c}1.51-2.56 \\
(1.84)\end{array}$ & $\begin{array}{c}0.56-2.48 \\
(1.34)\end{array}$ & $\begin{array}{c}0.39-0.86 \\
(0.65)\end{array}$ & $\begin{array}{l}42-661 \\
(218.1)\end{array}$ & $7-37(21.5)$ & $9-41(24.1)$ & $3-18(7.2)$ \\
\hline 5 & Dahod & $\begin{array}{c}11.5-14.8 \\
(13.3)\end{array}$ & $\begin{array}{c}0.42-1.04 \\
(0.78)\end{array}$ & $\begin{array}{c}1.49-2.96 \\
(1.87)\end{array}$ & $\begin{array}{c}0.65-2.82 \\
(1.60)\end{array}$ & $\begin{array}{c}0.49-0.68 \\
(0.58)\end{array}$ & $\begin{array}{l}30-483 \\
(199.8)\end{array}$ & $\begin{array}{l}19-78 \\
(35.1)\end{array}$ & $\begin{array}{l}20-46 \\
(29.9)\end{array}$ & $3-12(7.0)$ \\
\hline 6 & Vadodara & $\begin{array}{c}8.40-13.7 \\
(11.1)\end{array}$ & $\begin{array}{c}0.28-1.81 \\
(1.04)\end{array}$ & $\begin{array}{c}1.65-3.33 \\
(2.67) \\
\end{array}$ & $\begin{array}{c}0.84-2.33 \\
(1.38) \\
\end{array}$ & $\begin{array}{c}0.62-1.05 \\
(0.80)\end{array}$ & $\begin{array}{l}37-531 \\
(148.1) \\
\end{array}$ & $\begin{array}{c}41-162 \\
(78.9) \\
\end{array}$ & $\begin{array}{l}12-36 \\
(22.7) \\
\end{array}$ & $3-10(5.6)$ \\
\hline & Overall & $\begin{array}{c}8.40-16.5 \\
(12.7)\end{array}$ & $\begin{array}{c}0.28-2.13 \\
(1.04)\end{array}$ & $\begin{array}{c}1.45-3.33 \\
(2.17)\end{array}$ & $\begin{array}{c}0.56-3.87 \\
(1.71)\end{array}$ & $\begin{array}{c}0.39-1.62 \\
(0.73)\end{array}$ & $\begin{array}{l}28-663 \\
(220.8)\end{array}$ & $\begin{array}{l}7-162 \\
(45.9)\end{array}$ & $\begin{array}{c}9-68 \\
(26.5)\end{array}$ & $\begin{array}{c}3-21 \\
(6.91)\end{array}$ \\
\hline
\end{tabular}


Table.5 Nutrients uptake by one tonne of rice straw in different districts of middle Gujarat

\begin{tabular}{|c|c|c|c|c|c|c|c|c|c|c|}
\hline \multirow{2}{*}{$\begin{array}{l}\text { Sr. } \\
\text { No. }\end{array}$} & \multirow{2}{*}{ District } & \multicolumn{5}{|c|}{ Straw uptake $\left(\mathrm{kg} \mathrm{ha}^{-1}\right)$} & \multicolumn{4}{|c|}{ Straw uptake $\left(\mathrm{g} \mathrm{ha}^{-1}\right)$} \\
\hline & & $\mathbf{N}$ & $\mathbf{P}$ & $\mathbf{K}$ & $\mathbf{S}$ & $\mathbf{N a}$ & Fe & Mn & $\mathbf{Z n}$ & $\mathbf{C u}$ \\
\hline 1 & Ahmedabad & $\begin{array}{c}27.4-35.8 \\
(30.5) \\
\end{array}$ & $\begin{array}{c}0.50-2.23 \\
(1.97) \\
\end{array}$ & $\begin{array}{c}1.77-2.89 \\
(2.17) \\
\end{array}$ & $\begin{array}{c}1.00-5.77 \\
(3.18) \\
\end{array}$ & $\begin{array}{c}2.21-4.41 \\
(3.30) \\
\end{array}$ & $\begin{array}{l}72-487 \\
(259.4) \\
\end{array}$ & $\begin{array}{l}51-289 \\
(165.9) \\
\end{array}$ & $\begin{array}{l}11-46 \\
(29.6) \\
\end{array}$ & $2-10(5.5)$ \\
\hline 2 & Kheda & $\begin{array}{c}18.2-27.4 \\
(22.1) \\
\end{array}$ & $\begin{array}{c}0.93-1.93 \\
(1.22) \\
\end{array}$ & $\begin{array}{c}2.87-3.72 \\
(3.25)\end{array}$ & $\begin{array}{c}3.12-9.26 \\
(6.58) \\
\end{array}$ & $\begin{array}{c}2.26-3.08 \\
(2.63) \\
\end{array}$ & $\begin{array}{l}66-626 \\
(387.1) \\
\end{array}$ & $\begin{array}{c}66-513 \\
(189.1)) \\
\end{array}$ & $\begin{array}{c}4-35 \\
(20.5) \\
\end{array}$ & $5-18(9.6)$ \\
\hline 3 & Anand & $\begin{array}{c}24.9-35.8 \\
(29.2)\end{array}$ & $\begin{array}{c}0.70-1.34 \\
(0.96)\end{array}$ & $\begin{array}{c}3.09-4.01 \\
(3.57)\end{array}$ & $\begin{array}{c}1.94-4.84 \\
(3.61)\end{array}$ & $\begin{array}{c}1.39-2.57 \\
(1.86) \\
\end{array}$ & $\begin{array}{l}33-761 \\
(401.1)\end{array}$ & $\begin{array}{l}28-289 \\
(113.4)\end{array}$ & $\begin{array}{l}21-66 \\
(39.1) \\
\end{array}$ & $\begin{array}{c}3-17 \\
(10.1) \\
\end{array}$ \\
\hline 4 & Panchmahals & $\begin{array}{c}19.9-31.1 \\
(25.0)\end{array}$ & $\begin{array}{c}0.65-1.74 \\
(0.95)\end{array}$ & $\begin{array}{c}3.24-4.12 \\
(3.65)\end{array}$ & $\begin{array}{c}2.48-8.53 \\
(6.61) \\
\end{array}$ & $\begin{array}{c}1.28-3.28 \\
(2.45) \\
\end{array}$ & $\begin{array}{l}53-687 \\
(270.8) \\
\end{array}$ & $\begin{array}{l}67-684 \\
(254.2)\end{array}$ & $\begin{array}{l}15-55 \\
(29.9) \\
\end{array}$ & $1-11(5.2)$ \\
\hline 5 & Dahod & $\begin{array}{c}23.2-31.1 \\
(27.4)\end{array}$ & $\begin{array}{c}1.21-3.27 \\
(2.25)\end{array}$ & $\begin{array}{c}2.52-3.92 \\
(3.06)\end{array}$ & $\begin{array}{c}2.43-8.92 \\
(5.96)\end{array}$ & $\begin{array}{c}2.12-3.12 \\
(2.69) \\
\end{array}$ & $\begin{array}{c}181-869 \\
(398.7) \\
\end{array}$ & $\begin{array}{c}125-323 \\
(189.0) \\
\end{array}$ & $\begin{array}{l}17-48 \\
(32.3) \\
\end{array}$ & $3-18(7.8)$ \\
\hline 6 & Vadodara & $\begin{array}{c}22.4-26.0 \\
(24.2)\end{array}$ & $\begin{array}{c}1.30-2.29 \\
(1.87)\end{array}$ & $\begin{array}{c}4.02-4.87 \\
(4.41)\end{array}$ & $\begin{array}{c}5.67-10.12 \\
(7.59)\end{array}$ & $\begin{array}{c}0.75-1.68 \\
(1.32)\end{array}$ & $\begin{array}{c}151-614 \\
(299.8)\end{array}$ & $\begin{array}{c}113-629 \\
(316.3)\end{array}$ & $\begin{array}{l}15-65 \\
(31.0)\end{array}$ & $2-15(7.4)$ \\
\hline & Overall & $\begin{array}{c}18.2-35.8 \\
(26.4)\end{array}$ & $\begin{array}{c}0.50-3.27 \\
(1.54) \\
\end{array}$ & $\begin{array}{c}1.77-4.87 \\
(3.35) \\
\end{array}$ & $\begin{array}{c}1.00-10.12 \\
(5.59)\end{array}$ & $\begin{array}{c}0.75-4.41 \\
(2.38) \\
\end{array}$ & $\begin{array}{l}33-869 \\
(336.1) \\
\end{array}$ & $\begin{array}{l}28-684 \\
(204.6) \\
\end{array}$ & $\begin{array}{c}4-66 \\
(30.4) \\
\end{array}$ & $\begin{array}{c}1-18 \\
(7.6) \\
\end{array}$ \\
\hline
\end{tabular}

Table.6 Total uptake of nutrients by two tonne of rice produce in different districts of middle Gujarat

\begin{tabular}{|c|c|c|c|c|c|c|c|c|c|c|}
\hline \multirow{2}{*}{$\begin{array}{l}\text { Sr. } \\
\text { No. }\end{array}$} & \multirow{2}{*}{ District } & \multicolumn{5}{|c|}{ Total uptake $\left(\mathrm{kg} \mathrm{ha}^{-1}\right)$} & \multicolumn{4}{|c|}{ Total uptake $\left(\mathrm{g} \mathrm{ha}^{-1}\right)$} \\
\hline & & $\mathbf{N}$ & $\mathbf{P}$ & $\mathbf{K}$ & $\mathbf{S}$ & $\mathrm{Na}$ & Fe & Mn & $\mathbf{Z n}$ & $\mathbf{C u}$ \\
\hline 1 & Ahmedabad & $\begin{array}{c}38.6-49.6 \\
(43.7)\end{array}$ & $\begin{array}{c}1.01-3.72 \\
(2.67) \\
\end{array}$ & $\begin{array}{c}3.53-5.35 \\
(4.23) \\
\end{array}$ & $\begin{array}{c}3.36-8.68 \\
(5.91)\end{array}$ & $\begin{array}{c}3.21-5.54 \\
(4.32)\end{array}$ & $\begin{array}{c}125-652 \\
(429.0) \\
\end{array}$ & $\begin{array}{l}84-327 \\
(196.3) \\
\end{array}$ & $\begin{array}{l}34-66 \\
(48.1) \\
\end{array}$ & $5-20(10.9)$ \\
\hline 2 & Kheda & $\begin{array}{c}30.8-42.8 \\
(36.0)\end{array}$ & $\begin{array}{c}1.29-3.28 \\
(2.37)\end{array}$ & $\begin{array}{c}5.47-6.44 \\
(5.93)\end{array}$ & $\begin{array}{c}4.63-10.6 \\
(7.82)\end{array}$ & $\begin{array}{c}2.90-3.67 \\
(3.30)\end{array}$ & $\begin{array}{c}399-1537 \\
(975.2)\end{array}$ & $\begin{array}{c}182-603 \\
(318.4)\end{array}$ & $\begin{array}{l}27-77 \\
(51.3)\end{array}$ & $\begin{array}{l}10-32 \\
(17.3)\end{array}$ \\
\hline 3 & Anand & $\begin{array}{c}37.5-47.0 \\
(40.7)\end{array}$ & $\begin{array}{c}1.01-2.14 \\
(1.47)\end{array}$ & $\begin{array}{c}4.92-6.52 \\
(5.48)\end{array}$ & $\begin{array}{c}3.40-7.17 \\
(5.56)\end{array}$ & $\begin{array}{c}2.05-3.19 \\
(2.54)\end{array}$ & $\begin{array}{c}257-1479 \\
(792.2)\end{array}$ & $\begin{array}{c}58-339 \\
(161.9) \\
\end{array}$ & $\begin{array}{c}46-129 \\
(72.3) \\
\end{array}$ & $9-32(18.5)$ \\
\hline 4 & Panchmahals & $\begin{array}{c}33.0-43.7 \\
(38.7)\end{array}$ & $\begin{array}{c}0.96-2.69 \\
(1.57)\end{array}$ & $\begin{array}{c}4.82-6.27 \\
(5.49)\end{array}$ & $\begin{array}{c}4.66-11.01 \\
(7.95\end{array}$ & $\begin{array}{c}1.90-3.99 \\
(3.10)\end{array}$ & $\begin{array}{c}106-1052 \\
(488.9)\end{array}$ & $\begin{array}{l}83-691 \\
(275.7)\end{array}$ & $\begin{array}{l}30-80 \\
(54.0)\end{array}$ & $4-21(12.4)$ \\
\hline 5 & Dahod & $\begin{array}{c}36.7-44.8 \\
(40.7)\end{array}$ & $\begin{array}{c}1.86-3.83 \\
(2.93)\end{array}$ & $\begin{array}{c}4.40-5.67 \\
(4.94)\end{array}$ & $\begin{array}{c}3.37-11.40 \\
(7.56)\end{array}$ & $\begin{array}{c}2.73-3.69 \\
(3.27)\end{array}$ & $\begin{array}{c}268-1232 \\
(598.5)\end{array}$ & $\begin{array}{l}45-279 \\
(124.0)\end{array}$ & $\begin{array}{l}41-94 \\
(62.2) \\
\end{array}$ & $7-25(14.8)$ \\
\hline 6 & Vadodara & $\begin{array}{c}32.2-38.1 \\
(35.3)\end{array}$ & $\begin{array}{c}2.11-3.64 \\
(2.92)\end{array}$ & $\begin{array}{c}5.93-9.36 \\
(3.68)\end{array}$ & $\begin{array}{c}6.65-11.01 \\
(8.97)\end{array}$ & $\begin{array}{c}1.50-2.67 \\
(2.11)\end{array}$ & $\begin{array}{c}261-692 \\
(447.9)\end{array}$ & $\begin{array}{c}181-715 \\
(395.2)\end{array}$ & $\begin{array}{l}28-89 \\
(53.7) \\
\end{array}$ & $6-25(13.0)$ \\
\hline & Overall & $\begin{array}{c}30.8-60.8 \\
(41.05)\end{array}$ & $\begin{array}{c}0.96-4.2 \\
(2.33) \\
\end{array}$ & $\begin{array}{c}3.53-9.36 \\
(4.95)\end{array}$ & $\begin{array}{c}3.36-11.4 \\
(7.29)\end{array}$ & $\begin{array}{c}1.5-5.5 \\
(3.1) \\
\end{array}$ & $\begin{array}{c}106-1787 \\
(645.0)\end{array}$ & $\begin{array}{l}45-715 \\
(245.2)\end{array}$ & $\begin{array}{c}27-129 \\
(56.9)\end{array}$ & 4-32 (14.4) \\
\hline
\end{tabular}


Table.7 Ratio between silicon, major, secondary and micronutrients nutrients in rice grain in different districts of middle Gujarat

\begin{tabular}{|c|c|c|c|c|c|c|c|c|c|c|}
\hline \multirow{2}{*}{$\begin{array}{l}\text { Sr. } \\
\text { No }\end{array}$} & \multirow[t]{2}{*}{ District } & \multicolumn{9}{|c|}{$\mathrm{Si}$ : Nutrients ratio in grain } \\
\hline & & Si: $\mathbf{N}$ & Si: $\mathbf{P}$ & Si: K & Si: $\mathbf{S}$ & Si: Na & Si: Fe & Si: Mn & Si: Zn & Si: $\mathrm{Cu}$ \\
\hline 1 & Ahmedabad & $\begin{array}{c}1.01-3.69 \\
(1.81)\end{array}$ & $\begin{array}{c}6.3-60.6 \\
(23.9)\end{array}$ & $\begin{array}{c}4.4-28.3 \\
(12.0)\end{array}$ & $\begin{array}{c}3.8-24.2 \\
(9.2)\end{array}$ & $\begin{array}{c}7.5-95.1 \\
(26.8)\end{array}$ & $\begin{array}{c}89-958 \\
(405)\end{array}$ & $\begin{array}{c}201-1426 \\
(729)\end{array}$ & $\begin{array}{c}392-2604 \\
(1265)\end{array}$ & $\begin{array}{c}1308-14971 \\
(4986)\end{array}$ \\
\hline 2 & Kheda & $\begin{array}{c}2.61-4.58 \\
(3.54)\end{array}$ & $\begin{array}{c}16.8-124.0 \\
(53.7)\end{array}$ & $\begin{array}{c}13.4-23.5 \\
(18.3)\end{array}$ & $\begin{array}{c}12.4-30.7 \\
(21.5)\end{array}$ & $\begin{array}{c}37.2-59.6 \\
(56.8)\end{array}$ & $\begin{array}{c}43-304 \\
(117)\end{array}$ & $\begin{array}{c}213-837 \\
(407)\end{array}$ & $\begin{array}{c}718-2743 \\
(1789)\end{array}$ & $\begin{array}{c}2817-9642 \\
(6802)\end{array}$ \\
\hline 3 & Anand & $\begin{array}{c}1.22-4.85 \\
(2.86)\end{array}$ & $\begin{array}{c}13.2-79.7 \\
(37.4)\end{array}$ & $\begin{array}{c}6.1-24.9 \\
(17.3)\end{array}$ & $\begin{array}{c}6.1-42.8 \\
(19.1)\end{array}$ & $\begin{array}{c}20.2-77.7 \\
(48.4)\end{array}$ & $\begin{array}{c}22-514 \\
(166)\end{array}$ & $\begin{array}{c}172-1542 \\
(786)\end{array}$ & $\begin{array}{c}279-2006 \\
(1131) \\
\end{array}$ & $\begin{array}{c}1308-9477 \\
(4779) \\
\end{array}$ \\
\hline 4 & Panchmahals & $\begin{array}{c}1.01-2.55 \\
(1.56)\end{array}$ & $\begin{array}{c}10.3-43.6 \\
(21.1)\end{array}$ & $\begin{array}{c}6.9-17.1 \\
(11.7)\end{array}$ & $\begin{array}{c}7.3-51.0 \\
(19.3)\end{array}$ & $\begin{array}{c}19.4-62.6 \\
(33.6)\end{array}$ & $\begin{array}{c}30-595 \\
(182)\end{array}$ & $\begin{array}{l}395-1471 \\
(825)\end{array}$ & $\begin{array}{c}260-3165 \\
(1080)\end{array}$ & $\begin{array}{c}969-8915 \\
(3700)\end{array}$ \\
\hline 5 & Dahod & $\begin{array}{c}1.00-2.08 \\
(1.36)\end{array}$ & $\begin{array}{c}13.8-39.3 \\
(24.4)\end{array}$ & $\begin{array}{c}4.7-15.0 \\
(9.9)\end{array}$ & $\begin{array}{c}5.9-25.4 \\
(13.4)\end{array}$ & $\begin{array}{c}23.1-48.8 \\
(31.3)\end{array}$ & $\begin{array}{c}33-446 \\
(164)\end{array}$ & $\begin{array}{l}215-1005 \\
(587)\end{array}$ & $\begin{array}{c}433-1078 \\
(622)\end{array}$ & $\begin{array}{c}1421-6299 \\
(2916)\end{array}$ \\
\hline 6 & Vadodara & $\begin{array}{c}1.70-5.69 \\
(3.20)\end{array}$ & $\begin{array}{c}11.5-108.3 \\
(41.7)\end{array}$ & $\begin{array}{c}6.8-23.0 \\
(13.3)\end{array}$ & $\begin{array}{c}10.9-45.8 \\
(26.6)\end{array}$ & $\begin{array}{c}24.9-72.0 \\
(44.3)\end{array}$ & $\begin{array}{c}45-934 \\
(387)\end{array}$ & $\begin{array}{c}191-788 \\
(472)\end{array}$ & $\begin{array}{c}646-3616 \\
(1705)\end{array}$ & $\begin{array}{c}2326-11570 \\
(6546)\end{array}$ \\
\hline & Overall & $\begin{array}{c}1.00-5.69 \\
(2.37)\end{array}$ & $\begin{array}{c}\text { 6.3- 124.0 } \\
(33.7)\end{array}$ & $\begin{array}{c}4.4-28.3 \\
(13.0)\end{array}$ & $\begin{array}{c}\text { 3.8-51.0 } \\
(20.3)\end{array}$ & $\begin{array}{c}7.5-95.1 \\
(41.4)\end{array}$ & $\begin{array}{c}\text { 22-958 } \\
(237)\end{array}$ & $\begin{array}{c}172-1542 \\
(634)\end{array}$ & $\begin{array}{c}260-3616 \\
(1265)\end{array}$ & 969-14971 (4955) \\
\hline
\end{tabular}

Table.8 Ratio between silicon, major, secondary and micronutrients nutrients in rice straw in different districts of middle Gujarat

\begin{tabular}{|c|c|c|c|c|c|c|c|c|c|c|}
\hline \multirow{2}{*}{$\begin{array}{l}\text { Sr. } \\
\text { No }\end{array}$} & \multirow[t]{2}{*}{ District } & \multicolumn{9}{|c|}{ Si : Nutrients ratio in straw } \\
\hline & & Si: $\mathbf{N}$ & Si: $\mathbf{P}$ & Si: K & Si: $\mathbf{S}$ & $\mathrm{Si}: \mathbf{N a}$ & Si: Fe & Si: Mn & Si: Zn & Si: $\mathbf{C u}$ \\
\hline 1 & Ahmedabad & $\begin{array}{c}1.06-2.19 \\
(1.54)\end{array}$ & $\begin{array}{c}21.7-111.2 \\
(53.1)\end{array}$ & $\begin{array}{c}19.0-46.2 \\
(30.1)\end{array}$ & $\begin{array}{c}7.31-28.72 \\
(15.5)\end{array}$ & $\begin{array}{c}14.0-33.5 \\
(20.0)\end{array}$ & $\begin{array}{c}116-989 \\
(308)\end{array}$ & $\begin{array}{c}219-783 \\
(426)\end{array}$ & $\begin{array}{c}1156-4748 \\
(2333)\end{array}$ & $\begin{array}{c}5233-18702 \\
(12068)\end{array}$ \\
\hline 2 & Kheda & $\begin{array}{c}2.10-4.10 \\
(2.97)\end{array}$ & $\begin{array}{c}31.3-90.8 \\
(55.9)\end{array}$ & $\begin{array}{c}12.2-31.8 \\
(20.3)\end{array}$ & $\begin{array}{c}4.8-30.4 \\
(11.2)\end{array}$ & $\begin{array}{c}19.0-39.8 \\
(24.9)\end{array}$ & $\begin{array}{c}59-263 \\
(113)\end{array}$ & $\begin{array}{c}164-947 \\
(471)\end{array}$ & $\begin{array}{c}1655-9477 \\
(3757)\end{array}$ & $\begin{array}{c}3214-11725 \\
(7407)\end{array}$ \\
\hline 3 & Anand & $\begin{array}{c}1.08-2.45 \\
(1.87)\end{array}$ & $\begin{array}{c}30.4-85.1 \\
(58.2)\end{array}$ & $\begin{array}{c}9.6-20.2 \\
(15.2)\end{array}$ & $\begin{array}{c}9.6-25.1 \\
(15.5)\end{array}$ & $\begin{array}{c}16.4-39.2 \\
(29.6)\end{array}$ & $\begin{array}{c}41-453 \\
(176)\end{array}$ & $\begin{array}{c}168-1595 \\
(629)\end{array}$ & $\begin{array}{c}640-3277 \\
(1536)\end{array}$ & $\begin{array}{c}2856-12016 \\
(6143)\end{array}$ \\
\hline 4 & Panchmahals & $\begin{array}{c}1.52-4.24 \\
(2.85)\end{array}$ & $\begin{array}{c}21.6-72.1 \\
(50.4)\end{array}$ & $\begin{array}{c}10.4-26.6 \\
(19.2)\end{array}$ & $\begin{array}{c}5.9-20.3 \\
(11.3)\end{array}$ & $\begin{array}{c}13.9-62.7 \\
(31.1)\end{array}$ & $\begin{array}{c}81-997 \\
(432)\end{array}$ & $\begin{array}{c}95-1280 \\
(479)\end{array}$ & $\begin{array}{c}771-4850 \\
(2673)\end{array}$ & $\begin{array}{c}4123-23183 \\
(13049)\end{array}$ \\
\hline 5 & Dahod & $\begin{array}{c}1.42-2.99 \\
(2.04)\end{array}$ & $\begin{array}{c}15.6-43.3 \\
(26.5)\end{array}$ & $\begin{array}{c}13.4-28.2 \\
(18.3)\end{array}$ & $\begin{array}{c}5.5-19.2 \\
(10.3)\end{array}$ & $\begin{array}{c}13.9-27.7 \\
(20.8)\end{array}$ & $\begin{array}{c}48-406 \\
(173)\end{array}$ & $\begin{array}{c}223-1126 \\
(679)\end{array}$ & $\begin{array}{c}1060-3181 \\
(1859)\end{array}$ & $\begin{array}{c}2326-16958 \\
(8462)\end{array}$ \\
\hline 6 & Vadodara & $\begin{array}{c}1.56-4.32 \\
(2.82)\end{array}$ & $\begin{array}{c}18.9-54.5 \\
(36.6)\end{array}$ & $\begin{array}{c}8.7-24.4 \\
(15.4)\end{array}$ & $\begin{array}{c}4.3-17.3 \\
(9.3)\end{array}$ & $\begin{array}{c}26.5-90.2 \\
(53.2)\end{array}$ & $\begin{array}{c}108-470 \\
(251)\end{array}$ & $\begin{array}{c}66-723 \\
(303)\end{array}$ & $\begin{array}{c}662-5892 \\
(2551)\end{array}$ & $\begin{array}{c}1780-17675 \\
(10055)\end{array}$ \\
\hline & Overall & $\begin{array}{c}1.06-4.32 \\
(2.35)\end{array}$ & $\begin{array}{c}15.6-111.2 \\
(46.8)\end{array}$ & $\begin{array}{c}8.7-46.2 \\
(19.7)\end{array}$ & $\begin{array}{c}4.3-61.6 \\
(14.0)\end{array}$ & $\begin{array}{c}13.9-90.2 \\
(29.9)\end{array}$ & $\begin{array}{c}41-989 \\
(242)\end{array}$ & $\begin{array}{c}66-1595 \\
(498)\end{array}$ & $\begin{array}{c}640-9477 \\
(2451)\end{array}$ & $\begin{array}{c}\text { 1780-23183 } \\
(9530)\end{array}$ \\
\hline
\end{tabular}


Table.9 Correlation coefficients (r) between silicon content in grain with other nutrient contents of rice

\begin{tabular}{|c|c|c|c|c|c|c|}
\hline \multirow{2}{*}{ Grain Content } & \multicolumn{6}{|c|}{ Districts } \\
\hline & Ahmedabad & Kheda & Anand & Panchmahals & Dahod & Vadodara \\
\hline $\mathbf{N}(\%)$ & $0.551 * *$ & $-0.339 * *$ & $0.237 * *$ & $0.343^{* *}$ & $0.378 * *$ & $-0.182^{*}$ \\
\hline $\mathbf{P}(\%)$ & -0.126 & -0.061 & -0.130 & $0.419 * *$ & 0.167 & -0.161 \\
\hline K $(\%)$ & $0.187 *$ & -0.058 & 0.016 & $0.288 * *$ & -0.158 & -0.011 \\
\hline S (\%) & $0.198 *$ & -0.117 & $-0.238 * *$ & 0.086 & -0.038 & 0.078 \\
\hline $\mathrm{Na}(\%)$ & -0.141 & -0.034 & $0.280 * *$ & -0.069 & 0.031 & -0.088 \\
\hline Fe (ppm) & -0.092 & $-0.192^{*}$ & -0.154 & -0.116 & $-0.676 * *$ & $-0.221^{*}$ \\
\hline Mn (ppm) & 0.067 & 0.127 & $0.179 *$ & 0.044 & $0.506 * *$ & $0.248 * *$ \\
\hline Zn (ppm) & $0.474 * *$ & 0.082 & -0.140 & 0.162 & $0.275^{* *}$ & $-0.199 *$ \\
\hline $\mathrm{Cu}$ (ppm) & -0.028 & 0.068 & -0.144 & -0.033 & $0.261 * *$ & -0.057 \\
\hline
\end{tabular}

Table.10 Correlation coefficient (r) between silicon content in straw with other nutrient contents of rice

\begin{tabular}{|c|c|c|c|c|c|c|}
\hline \multirow{2}{*}{$\begin{array}{l}\text { Straw } \\
\text { Content }\end{array}$} & \multicolumn{6}{|c|}{ Districts } \\
\hline & Ahmedabad & Kheda & Anand & Panchmahals & Dahod & Vadodara \\
\hline $\mathbf{N}(\%)$ & $-0.290 * *$ & $0.430 * *$ & -0.153 & 0.002 & 0.069 & $-0.528 * *$ \\
\hline $\mathbf{P}(\%)$ & $0.317 * *$ & -0.171 & 0.112 & $-0.245^{* *}$ & 0.174 & 0.138 \\
\hline $\mathbf{K}(\%)$ & $-0.318 * *$ & -0.124 & $0.229 *$ & 0.148 & 0.051 & $-0.250 * *$ \\
\hline S (\%) & $-0.383^{* *}$ & $-0.329 * *$ & $0.255^{* *}$ & 0.166 & 0.125 & $-0.626 * *$ \\
\hline $\mathrm{Na}(\%)$ & $-0.179 *$ & $0.319 * *$ & $0.219 *$ & -0.059 & 0.123 & 0.095 \\
\hline Fe (ppm) & $0.272 * *$ & 0.103 & $-0.361 * *$ & $-0.340 * *$ & $-0.310 * *$ & 0.148 \\
\hline Mn (ppm) & $0.399 * *$ & 0.001 & -0.082 & $-0.182 *$ & $-0.623 * *$ & $-0.257 * *$ \\
\hline Zn (ppm) & $-0.444 * *$ & -0.104 & -0.038 & $-0.184 *$ & -0.098 & -0.115 \\
\hline $\mathrm{Cu}$ (ppm) & -0.173 & $0.210^{*}$ & 0.151 & $-0.262 * *$ & 0.072 & 0.160 \\
\hline
\end{tabular}

At national level, field experiment conducted at Varanasi centre (Singh et al., 2005) showed increased $\mathrm{N}, \mathrm{P}, \mathrm{K}$ concentration and uptake due to silicon application @ $180 \mathrm{~kg} \mathrm{ha}^{-1}$ full as basal. Similar results were found that Sudhakar et al., (2006), increasing the fertility level significantly improved nitrogen, phosphorus and silicon uptake by grain and straw, whereas significant improvement in potassium uptake by grain and straw was recorded. The increase in nutrient uptake with the increase in fertility levels could be attributed to the better availability of nutrients and their transport to the plant from the soil, as the nutrient application leads to its enhanced uptake by plant (Rani et al., 1997).
The similar findings have been also reported by Meshram et al., (2015), the higher nutrient uptake was mainly due to higher biological (straw + grain) yield.

\section{Ratio of silicon with other nutrients in rice}

The results on silicon and other nutrients ratio in rice grain and straw was reported in Table 7 and 8 . The ratio of silicon and other nutrient contents in rice grain indicated that amongst $\mathrm{N}, \mathrm{P}, \mathrm{K}, \mathrm{S}$ and $\mathrm{Na}$, the higher ratio was observed in $\mathrm{Si}$ : $\mathrm{P}$ indicating lower content of $\mathrm{P}$ in comparison with silicon. The ascending order of ratio of $\mathrm{Si}$ with nutrients was: $\mathrm{N}>\mathrm{K}$ $>\mathrm{S}>\mathrm{Na}>\mathrm{P}$ in rice grain. 
The lower content of $\mathrm{P}$ may be attributed the low status of available $\mathrm{P}_{2} \mathrm{O}_{5}$ and dominancy of $\mathrm{Si}$ at root level entry on phosphorous (Table 7). Similar results were also observed in case of straw that lower $\mathrm{P}$ content in straw followed by $\mathrm{Na}, \mathrm{K}, \mathrm{S}$ and $\mathrm{N}$. The soils of the region having more per cent of sufficiency in available $\mathrm{K}_{2} \mathrm{O}$ and $\mathrm{S}$ resulted in high content of respective nutrients in rice. The ratio also indicated that $\mathrm{Si}$ accumulation in rice straw was almost 2, 47, 20, 14 and 30 times higher than $\mathrm{N}, \mathrm{P}, \mathrm{K}, \mathrm{S}$ and $\mathrm{Na}$ content, respectively (Table 8) (Malav et al., 2015). The overall Si: micronutrients $(\mathrm{Fe}, \mathrm{Mn}, \mathrm{Zn}, \mathrm{Cu})$ ratio in rice grain was 237, 634, 1265 and 4955 indicates the high contents of $\mathrm{Fe}$ and $\mathrm{Mn}$ as compared to $\mathrm{Zn}$ and $\mathrm{Cu}$ (Table 7). The soils of rice growing areas having medium to high status of available $\mathrm{Fe}$ and $\mathrm{Mn}$ attributed the sufficient supply of $\mathrm{Fe}$ and $\mathrm{Mn}$ during crop growth. Similar results were observed of micronutrients ratio with silicon in rice straw. The ascending order of ratio of $\mathrm{Si}$ with micronutrients was $\mathrm{Fe}>\mathrm{Mn}>\mathrm{Zn}>\mathrm{Cu}$ indicates the higher accumulation of $\mathrm{Fe}$ in straw as compared to $\mathrm{Mn}, \mathrm{Zn}$ and $\mathrm{Cu}$ (Table 8). According to Ma and Takahashi (1991), the beneficial effect of silicon may be attributed to a higher $\mathrm{P}$ : Mn ratio in the shoot due to the decreased $\mathrm{Mn}$ and Fe uptake and thus indirectly to improve $\mathrm{P}$ utilization within the rice plants. They had concluded that the interaction between phosphorus and silicon was indirect in phosphorus deficient soil.

The results of wider values for ratios of $\mathrm{Si}$ with nutrients ( $\mathrm{Fe}, \mathrm{Mn}, \mathrm{Zn}$ and $\mathrm{Cu}$ ) can be supported by Cheng (1982) reported that addition of silicon to the culture solution at the rate of $75 \mathrm{ppm}$ silicon, decreased the accumulation of $\mathrm{Mn}, \mathrm{Cu}, \mathrm{Fe}, \mathrm{Zn}, \mathrm{N}, \mathrm{P}$ and transpiration rate but increased $\mathrm{Ca}, \mathrm{Mg}, \mathrm{Si}$ and carbohydrate content of the plant. Further, Park (1976) reported that the response of rice to $\mathrm{SiO}_{2}$ application was also related to the supply of other nutrients in the top soil (N, P, K, Ca and $\mathrm{Mg}$ ) and it seems if a proper balance of nutrients were required if we obtain the maximum yield of rice (i.e. $\mathrm{SiO}_{2} / \mathrm{N}, \mathrm{K}_{2} \mathrm{O} / \mathrm{N}, \mathrm{SiO}_{2} / \mathrm{K}_{2} \mathrm{O}$ ratios in the straw should be around 11, 1.5 and 7.4, respectively). The $\mathrm{SiO}_{2}$ material usually contains $\mathrm{Ca}$ and $\mathrm{Mg}$ and these bases influence the uptake of $\mathrm{K}$ and minor elements such as $\mathrm{Zn}$ and $\mathrm{B}$ probably owing to antagonism and the change in the availability of these elements in the topsoil (Nayak et al., 2014). These facts lead to the concept of the maintenance of multifactor balance in rice soil management if high yields were to be obtained. Similar results also reported by Mongia and Chhabra (2001).

\section{Relationship of silicon and other nutrients content in rice grain}

The district wise correlation study between silicon content in grain with other important nutrient contents in rice revealed that the rice grain silicon gave significant correlation with all other nutrient content (Table 9). In case of Ahmedabad district, coefficient of correlation between the $\mathrm{N}$ and $\mathrm{Zn}$ contents in rice grain with silicon content showed highly significant positive $\left(\mathrm{r}=0.551^{* *}\right.$ and $\mathrm{r}=0.474 * *$, respectively); and $\mathrm{K}$ and $\mathrm{S}$ content showed significant positive $\left(\mathrm{r}=0.187^{*}\right.$ and $\mathrm{r}=$ $0.198^{*}$, respectively) relationship. Whereas, $\mathrm{P}, \mathrm{Na}, \mathrm{Fe}$ and $\mathrm{Cu}$ content in grain showed negative relationship but the results where non-significant when they all were correlated with content of silicon in grain.

In Kheda district, the grain $\mathrm{N}$ content showed the reverse correlation than Ahmedabad district that $\mathrm{N}$ and $\mathrm{Fe}$ showed the highly significant $(\mathrm{r}=-0.339 * *)$ and significant $(\mathrm{r}=$ 0.192), negative correlation, respectively when correlated with silicon content in grain and other nutrients content showed the nonsignificant results. The relationship between silicon content in grain with other nutrients in 
Anand district was somewhat different than other districts as $\mathrm{N}$ and $\mathrm{Na}$ showed highly positive $\left(\mathrm{r}=0.237^{* *}\right.$ and $\left.\mathrm{r}=0.280^{* *}\right)$ correlation, while Mn content gave significant positive $(r=0.179 *)$ relationship. Only $\mathrm{S}$ content in grain resulted significantly negatively correlated $\left(r=-0.238^{*}\right)$ with silicon content in grain.

In Panchmahal district, $\mathrm{N}, \mathrm{P}$ and $\mathrm{K}$ content in grain $\left(\mathrm{r}=0.343 * *, 0.419 * *\right.$ and $0.288^{* *}$, respectively) showed highly positive significant relationship with silicon content in grain, whereas, other nutrients content showed nonsignificant associationship with silicon. In case of Dahod district, the value of correlation coefficient (r) rather lesser than other districts as $\mathrm{N}, \mathrm{Mn}, \mathrm{Zn}$ and $\mathrm{Cu}$ content showed highly significant positive correlationship $\left(\mathrm{r}=0.378^{* *}\right.$, $0.506^{* *}, 0.275^{* *}$ and $0.261^{* *}$, respectively). Whereas, $\mathrm{Fe}$ content in grain had highly negative significant relationship $\left(\mathrm{r}=-0.676^{* *}\right)$ with silicon content in grain. The Mn content in grain $\left(\mathrm{r}=0.248^{* *}\right)$ was highly significant positively correlated in Vadodara district, while in context $\mathrm{N}, \mathrm{Fe}$ and $\mathrm{Zn}(\mathrm{r}=-0.182 *,-0.221 *$ and $-0.199 *$, respectively) were gave significantly negative correlationship with silicon. Dean and Rubins, (1947) all in one line reported that ability of silicate ion to displace the phosphate ion from the adsorbed conditions in the soil thus increasing the availability of phosphorus. But in contrast, application of silicate was reported to decrease the phosphorus fixing capacity of some highly weathered volcanic ash soils (Iwata and Baba 1962). He reported that rate of silicon release from clay mineral was enhanced immediately after application of $\mathrm{P}$.

\section{Relationship of silicon and other nutrients content in rice straw}

The district wise correlation coefficient between silicon content in straw with other important nutrient contents in rice also worked out and given in Table 10. The results indicated that silicon content in rice straw had significant correlation with all other nutrient content (Table
10). The total $\mathrm{P}, \mathrm{Fe}$ and $\mathrm{Mn}$ content in rice straw showed highly significant positive associationship $(\mathrm{r}=0.317 * *, 0.272 * *$ and $0.399 * *$, respectively) and $\mathrm{N}, \mathrm{K}, \mathrm{S}$ and $\mathrm{Zn}$ showed highly significant negative $(\mathrm{r}=$ $0.290^{* *}, \quad-0.318^{* *}, \quad-0.383^{* *}$ and $-0.444^{* *}$, respectively) impact in Ahmedabad district. While, Na showed the significant negative $(\mathrm{r}=$ 0.179 ) impact, when they were correlated with silicon content in straw.

In Kheda district, the $\mathrm{N}, \mathrm{Na}$ and $\mathrm{Cu}$ showed the reverse correlation than Ahmedabad district. as $\mathrm{N}$ and $\mathrm{Na}$ showed the highly positive significant $(\mathrm{r}=0.430 * *$ and $0.419 * *$, respectively) relationship and $\mathrm{Cu}$ showed the significant $(\mathrm{r}=$ $0.210 *$ ), positive correlation with Si content in straw. The total S content in straw gave highly significant negative correlation $(\mathrm{r}=0.329 * *)$ with silicon and other nutrient content showed the non-significant results. Silicon in rice straw in Anand district showed highly positive ( $\mathrm{r}=$ $0.255^{* *}$ ) correlation, while $\mathrm{K}$ and $\mathrm{Na}$ gave significant positive $\left(\mathrm{r}=0.229^{*}\right.$ and $\left.0.219^{*}\right)$ relationship with $\mathrm{S}$ content in straw. The $\mathrm{Fe}$ content in straw only had highly significant negative correlation $(\mathrm{r}=-0.361 * *)$ with silicon content in straw.

In Panchmahal district, silicon content showed mostly negative correlation with $\mathrm{P}, \mathrm{Fe}$ and $\mathrm{Cu}(\mathrm{r}$ $=-0.245^{* *}, \quad-0.340 * *$ and $-0.262 * *$, respectively) content in straw. Whereas, $\mathrm{Mn}$ and $\mathrm{Zn}$ content had the significant negative relationship $\left(\mathrm{r}=-0.182^{*}\right.$ and $-0.184^{*}$, respectively) with silicon content. In case of Dahod district, the Fe and Mn content in straw indicated highly significant negative correlationship $(\mathrm{r}=-0.310 * *$ and $-0.623 * *$, respectively). While other nutrients content showed non-significant correlationship with silicon. In Vadodara districts, correlation coefficient between $\mathrm{N}, \mathrm{K}, \mathrm{S}$ and $\mathrm{Mn}$ content $(\mathrm{r}=$ $-0.528 * *,-0.250 * *,-0.626 * *$ and $-0.257 * *$, respectively) and silicon content gave significantly negative correlationship and it was non-significant with other nutrient contents in straw. Slag might neutralise the soil acidity with the formation of silicic acid and could thus 
diminish the solubility of such elements as Mn, $\mathrm{Fe}$ and $\mathrm{Al}$ (Okuda and Takahashi, 1962; Ayers, 1966). Therefore formation of silicic acid somehow in soils could be the reason for negative results of $\mathrm{Si}$ with $\mathrm{Fe}$ and $\mathrm{Mn}$ contents of soils of middle Gujarat.

The silicon content of rice grain collected from the region ranged from 1.20 to 5.99 per cent and the rice straw $\mathrm{Si}$ content ranged from 3.52 to 9.80 per cent of different districts of middle Gujarat region. The total uptake of $\mathrm{Si}$ by rice was $88,114,87,91,73$ and $102 \mathrm{~kg} \mathrm{ha}^{-1}$ and the uptake of Si was nearly 2 to 45 times more as compared to N, P, K and S uptake by rice. The ratio of silicon and other nutrient contents in rice grain and straw indicated that amongst $\mathrm{N}$, $\mathrm{P}, \mathrm{K}, \mathrm{S}$ and $\mathrm{Na}$, the higher ratio was observed in $\mathrm{Si}$ : $\mathrm{P}$ indicating lower content of $\mathrm{P}$ in comparison with silicon.

\section{Future Thrust}

Extensive survey of intensively cultivated rice ecosystem must be conducted to identify soil, water and plant silicon status. Identification of efficient $\mathrm{Si}$ accumulating paddy cultivars is desired. Standardization of cheap silicon fertilizer sources is very important as per Indian scenario for sustaining the agricultural productivity.

\section{References}

Anonymous 2009. Directorate of Economics and Statistics, Department of Agriculture and Cooperation.

Ayres 1966. Calcium silicate slag as a growth stimulant for sugarcane on low silicon soil. Soil Sci., 101 (3): 216 - 227.

Chaudhary BI and Cornfield AH 1966. The determination of total sulphur in soils and plant material, Analyst, 91, 1085, 528.

Cheng BT 1982. Some significant functions of silicon to higher plants. J. Plant Nutr. 5: 1345-1353

Dai W, Zhang K, Duan B, Sun C, Zheng K, Cai $\mathrm{R}$ and Zhuang J 2005. Rapid Determination of Silicon Content in Rice.
Rice Science, 12 (2): 145-147.

Dean LA and Rubins EJ 1947. Anion exchange in soils. I. Exchangeable phosphorus and the anion exchange capacity. Soil Sci. 63(5): 377-387.

Elawad SH and Green VE 1979. Silicon and the rice plant environment: A review of recent research. II Riso 28: 235-253.

Epstein E1994. The anomaly of silicon in plant biology. Proc. Natl. Acad. Sci. 91:11-17.

Ganaie AQ, Bhat ZA, Padder SA and Bashir I 2015. Effect of long-term application of integrated nutrient management on crop yield and nutrient uptake under ricewheat cropping sequence. The Ecoscan. 9: 277-283.

Imaizumi $\mathrm{K}$ and Yoshida S 1958. Edaphological studies on silicon supplying power of rice soils. Bull. Natl. Inst. Agric. Sci. (Jpn) B 8: 261-304.

Islam A and Saha RC 1969. Effects of silicon on the chemical composition of rice plants. Plant and soil XXX no. 3: 446-458.

Iwata Y and Baba I 1962. Studies on the varietal adaptability for heavy manuring in rice effect of silica upon the adaptability of the rice plant for heavy manuring in relation to photosynthesis. Proceeding Soil Science Society Japan. 30: 237-240.

Jackson ML 1973. Soil Chemical Analysis, Prentice Hall, New Delhi.

Kawaguchi K 1966. Tropical rice soils. Jpn. Agric. Res. Q. 1:7-11.

Korndorfer GH, Snyder GH, Ulloa $M$ and Datnoff LE 2001. Calibration of soil and plant silicon for paddy production. Journal of Plant Nutrition 24: 1071 1084.

Ma JF and Takahashi E 1989. Effect of silicic acid on phosphorus uptake by rice plant. Soil Science Plant Nutrition 35(2): 227234.

Ma JF and Takahashi E 1991. Availability of rice straw $\mathrm{Si}$ to rice plants. Soil Science Plant Nutrition 37(1): 111-116.

Malav JK, Patel KC and Sajid M 2015. Influence of silicon fertilization on yield and nutrients uptake ( $\mathrm{Si}, \mathrm{P}, \mathrm{K}, \mathrm{S} \& \mathrm{Na}$ ) of 
Rice (Oryza Sativa L.). The Ecoscan. 9(1\&2): 629-634.

Meshram MR, Dwivedi SK, Ransing DM and Pandey P 2015. Response of customized fertilizer on productivity, nutrient uptake and energy use of rice (Oryza Sativa L.). The Ecoscan. 9: 373-376.

Miyake Y 1993. Silica in soils and plants. Sci. Rep. Faculty of Okayama Univ. 81: 61-79 (In Japanese with English Summary).

Mongia AD and Chhabra R 2000. Silica and phosphate profiles of alkali soils following reclamation. J. Indian Society Soil Science 48: 33- 37.

Nayak T, Bajpai RK and Bachkaiya V 2014. Vertical distribution of potassium under organic and inorganic nutrient management in a Vertisol planted to rice. The Ecoscan. 8: 257-261.

Nayar PK, Misra AK and Patnaik S 1975. Rapid micro-determination of silicon in rice plant. Plant and Soil, 42: 491-494.

Nayar PK, Misra AK and Patnaik S 1977. Evaluation of silica - supplying power of soils for growing rice. Plant and Soil, 47: 487-494.

Okuda A and Takahashi E 1962. Effect of silicon supply on the injuries due to excessive amounts of $\mathrm{Fe}, \mathrm{Mn}, \mathrm{Cu}, \mathrm{As}, \mathrm{AI}$, Co of barley and rice plant. Soil Science Plant Nutrition 33: 1-8.

Park CS 1975. Practice of fertilizer application for rice under the shortage of chemical fertilizers in Korea (mimeo). Paper delivered at Seminar on 'Maintaining rice production in the face of shortage of chemical fertilizers". FFTC, Taipei, Taiwan.

Savant NK, Snyder GH and Datnoff LE1997. Silicon management and sustainable rice production. Adv. in Agron. 58: 151-199.

Singh K, Singh Y, Singh CS, Singh R, Singh KK and Singh AK 2005. Silicon nutrition in rice. Fertilizer news. 50(2): 41-48.

Singh KK, Singh K, Ragevendra S, Yogeshvar $S$ and Singh CS 2006. Response of Nitrogen and silicon levels on growth, yield attribute and nutrient uptake of rice (Oryza sativa L.). Oryza 43: 220-223.

Steel RG and Torrie JH 1982. Principles and procedures of statistics. McGraw Hill Book Company, New Delhi - 110001.

Takahashi E 1995. Uptake, mode and physiological functions of silica. Science Rice Plant 2: 58-71.

Takahashi E and Miyake Y 1977. Silica and plant growth. In "Proceeding of the International Seminar on Environment and Fertility Management in Intensive Agriculture" (SEFMIA), Tokyo, Japan, pp. 603-611.

Takijima YH, Wijayaratna MS and Soneviratne CJ 1970. Nutrient deficiency and physiological disease of lowland rice in Ceylon. III. Effect of silicate fertilizers and dolomite for increasing rice yield. Soil Science Plant Nutrition 16: 11-16.

Verma TS and Minhas RS 1989. Effect of iron and manganese interactions in rice yield and iron and manganese nutrition in Silicon treated and untreated soils. Soil Sci. 147: 107-115.

Yoshida S 1975. The physiology of silicon in rice. Technical Bulletin No. 25. Food Fertilizer Technology Centre, Taipei, Taiwan.

Yoshida S 1981. Fundamentals of rice crop science. International Rice Research Institute, Los Banos, Laguna, Philippines.

\section{How to cite this article:}

Patel, R.A., K.C. Patel and Malav, J.K. 2017. Status of Silicon in Rice (Oryza sativa L.) and its Correlation with Other Nutrients under Typic ustochrepts Soil. Int.J.Curr.Microbiol.App.Sci. 6(12): 2598-2611. doi: https://doi.org/10.20546/ijcmas.2017.612.301 